PROCEEDINGS OF THE

AMERICAN MATHEMATICAL SOCIETY

Volume 139, Number 11, November 2011, Pages 3929-3936

S 0002-9939(2011)10806-2

Article electronically published on March 10, 2011

\title{
MONOTONICITY AND ASYMPTOTICS OF ZEROS OF LAGUERRE-SOBOLEV-TYPE ORTHOGONAL POLYNOMIALS OF HIGHER ORDER DERIVATIVES
}

\author{
FRANCISCO MARCELLÁN AND FERNANDO R. RAFAELI
}

(Communicated by Walter Van Assche)

\begin{abstract}
In this paper we analyze the location of the zeros of polynomials orthogonal with respect to the inner product

$$
\langle p, q\rangle=\int_{0}^{\infty} p(x) q(x) x^{\alpha} e^{-x} d x+N p^{(j)}(0) q^{(j)}(0),
$$

where $\alpha>-1, N \geq 0$, and $j \in \mathbb{N}$. In particular, we focus our attention on their interlacing properties with respect to the zeros of Laguerre polynomials as well as on the monotonicity of each individual zero in terms of the mass $N$. Finally, we give necessary and sufficient conditions in terms of $N$ in order for the least zero of any Laguerre-Sobolev-type orthogonal polynomial to be negative.
\end{abstract}

\section{Introduction AND STATEMENT OF RESUlts}

Given a nontrivial probability measure $\mu$ supported in an infinite subset of the real line and a real number $c$, sequences of polynomials orthogonal with respect to the Sobolev inner product,

$$
\langle p, q\rangle=\int_{\mathbb{R}} p(x) q(x) d \mu(x)+N p^{(j)}(c) q^{(j)}(c),
$$

have been introduced in [1]. There, the authors obtain a higher order recurrence relation that such polynomials satisfy as well as second order linear differential equations of holonomic type assuming that the measure $\mu$ is semi-classical. The zero distribution of such polynomials when the support of $\mu$ is located in the positive real semi-axis and $c=0$ is analyzed in [12. There the author proves that the zeros of the polynomials of degree $n$ are real, simple, and at most one of them is outside $(0,+\infty)$. The location of these zeros is given in terms of the location of the zeros of the polynomials orthogonal with respect to the measures $d \mu$ and $x^{2} d \mu$.

Next, we will focus our attention on a canonical case of the above inner product related to the Laguerre weight. Indeed, let us consider the sequence of polynomials

Received by the editors November 25, 2009 and, in revised form, September 7, 2010.

2010 Mathematics Subject Classification. Primary 42C05; Secondary 33C47.

Key words and phrases. Laguerre orthogonal polynomials, Laguerre-Sobolev-type orthogonal polynomials, zeros, interlacing, monotonicity, asymptotics.

(C)2011 American Mathematical Society Reverts to public domain 28 years from publication 
$\left\{L_{n}^{(\alpha, N)}(x)\right\}_{n=0}^{\infty}$ orthogonal with respect to the Sobolev-type inner product

$$
\langle p, q\rangle=\int_{0}^{\infty} p(x) q(x) x^{\alpha} e^{-x} d x+N p^{(j)}(0) q^{(j)}(0),
$$

where $\alpha>-1, N \geq 0$, and $j \in \mathbb{N}$ as well as the Laguerre polynomials $\left\{L_{n}^{(\alpha)}(x)\right\}_{n=0}^{\infty}$, normalized as in Szegö's book [13, i.e., $L_{n}^{(\alpha)}(0)=\left(\begin{array}{c}n+\alpha \\ n\end{array}\right)$.

The case $j=1$ has attracted the interest of many researchers from many points of view. First, the analysis of the asymptotic properties of such orthogonal polynomials is done (see [1] and the survey paper [10]). Second, the study of analytic properties of their zeros such as interlacing with the zeros of Laguerre polynomials and monotonicity of each zero in terms of $N$, among others, are completely discussed in [4. Furthermore, the limit behavior of such zeros is also deduced in 10. Third, the study of spectral properties of these orthogonal polynomials in the sense that they are eigenfunctions of an infinite order linear differential operator which reduces to order $2 \alpha+8$ if $\alpha$ is a nonnegative integer number and $N>0$ is presented in [9. Finally, a second order linear differential equation of holonomic type that such polynomials satisfy is deduced in [7]. As an immediate consequence, an electrostatic interpretation of these zeros is given.

In recent years, some attention was paid to the asymptotic properties of these orthogonal polynomials when $j>1$. In particular, in [6] the authors obtain a Mehler-Heine formula for such polynomials as well as their outer relative asymptotics in terms of the standard Laguerre orthogonal polynomials. On the other hand, in 2] it is proved that these polynomials are eigenfunctions of an infinite order linear differential operator which reduces to order $2 \alpha+4+4 j$ if $\alpha$ is a nonnegative integer number and $N>0$. But, the analysis of their zeros in terms of $N$ remains an open problem. This is the aim of our contribution.

The structure of the manuscript is as follows. In Section 2, a connection formula for the sequences of standard Laguerre orthogonal polynomials and the LaguerreSobolev type orthogonal polynomials is given. Notice that this connection formula is quite different from the connection formula given in Theorem 3.1 of [12]. It will be very useful in the study of some analytic properties of the zeros of LaguerreSobolev-type orthogonal polynomials. Indeed, in Section 3 we deduce interlacing properties between the zeros of such sequences as well as locating the least zero of $L_{n}^{(\alpha, N)}(x)$ with respect to the interval $(0,+\infty)$ in terms of the mass $N$. Furthermore, we prove that each zero is a decreasing function of $N$ and we analyze its limit when $N$ tends to infinity as well as the speed of convergence. These results are new in the literature as far as we know. Some numerical examples allow us to check our results.

\section{Connection formula}

In the following, we adopt the convention $\left(\begin{array}{l}i \\ 0\end{array}\right):=1$ and $\left(\begin{array}{c}i \\ -j\end{array}\right):=0, i, j>0$.

Theorem 1. Let $\left\{L_{n}^{(\alpha, N)}(x)\right\}_{n=0}^{\infty}$ be the Laguerre-Sobolev-type orthogonal polynomials corresponding to the inner product (1.2). Then, for every $n>j$ and each $\alpha>-1$,

$$
L_{n}^{(\alpha, N)}(x)=\sum_{k=0}^{j+1} A_{n, k} L_{n-k}^{(\alpha+k)}(x),
$$


where

$$
\begin{aligned}
& A_{n, 0}=1+N \frac{\Gamma(j+1)}{\Gamma(\alpha+j+1)} \sum_{k=1}^{j+1}(-1)^{k+1}\left(\begin{array}{c}
n+\alpha \\
n-j-k
\end{array}\right)\left(\begin{array}{c}
n-k \\
j+1-k
\end{array}\right), \\
& A_{n, k}=(-1)^{k} N \frac{\Gamma(j+1)}{\Gamma(\alpha+j+1)}\left(\begin{array}{c}
n+\alpha \\
n-j
\end{array}\right)\left(\begin{array}{c}
n-k \\
j+1-k
\end{array}\right), \quad k=1, \ldots, j+1 .
\end{aligned}
$$

Proof. Let $\left\{L_{n}^{(\alpha, N)}(x)\right\}_{n=0}^{\infty}$ be the sequence of polynomials defined by (2.3) and (2.4). We shall prove the orthogonality of these polynomials with respect to the inner product (1.2). Denote by $\pi_{n}$ the set of polynomials of degree at most $n$. Let $p_{l+j+1}(x)=x^{j+1} q_{l}(x), l=0,1, \ldots, n-(j+2)$, where $q_{l}(x)$ is a polynomial of degree exactly $l$. Thus, the polynomials $1, x, \ldots, x^{j}$ and $p_{l+j+1}(x), l=0,1, \ldots, n-(j+2)$, constitute a basis in $\pi_{n-1}$. Then, for $n \geq j+2$,

$$
\begin{aligned}
\left\langle p_{l+j+1}, L_{n}^{(\alpha, N)}\right\rangle & =\int_{0}^{\infty} x^{j+1} q_{l}(x) L_{n}^{(\alpha, N)}(x) x^{\alpha} e^{-x} d x \\
& =\sum_{k=0}^{j+1} A_{n, k} \int_{0}^{\infty} x^{j+1-k} q_{l}(x) L_{n-k}^{(\alpha+k)}(x) x^{\alpha+k} e^{-x} d x \\
& =0
\end{aligned}
$$

because of the orthogonality property of the classical Laguerre polynomials. Now, we define the integral $I_{k, i}$ by (see [8, (3.3)])

$$
I_{k, i}=\int_{0}^{\infty} L_{n-i}^{(\alpha+i)}(x) x^{\alpha+k} e^{-x} d x=\left(\begin{array}{c}
n-k-1 \\
n-i
\end{array}\right) \Gamma(\alpha+k+1) .
$$

Thus,

$$
\begin{aligned}
\left\langle 1, L_{n}^{(\alpha, N)}\right\rangle= & \int_{0}^{\infty} L_{n}^{(\alpha, N)}(x) x^{\alpha} e^{-x} d x \\
= & A_{n, 0} \cdot 0+A_{n, 1} \cdot I_{0,1}+A_{n, 2} \cdot I_{0,2}+\cdots+A_{n, j} \cdot I_{0, j}+A_{n, j+1} \cdot I_{0, j+1}, \\
\left\langle x, L_{n}^{(\alpha, N)}\right\rangle= & \int_{0}^{\infty} x L_{n}^{(\alpha, N)}(x) x^{\alpha} e^{-x} d x \\
= & A_{n, 0} \cdot 0+A_{n, 1} \cdot 0+A_{n, 2} \cdot I_{1,2}+\cdots+A_{n, j} \cdot I_{1, j}+A_{n, j+1} \cdot I_{1, j+1}, \\
& \vdots \\
\left\langle x^{j-1}, L_{n}^{(\alpha, N)}\right\rangle= & \int_{0}^{\infty} x^{j-1} L_{n}^{(\alpha, N)}(x) x^{\alpha} e^{-x} d x \\
= & A_{n, 0} \cdot 0+A_{n, 1} \cdot 0+A_{n, 2} \cdot 0+\cdots+A_{n, j} \cdot I_{j-1, j}+A_{n, j+1} \cdot I_{j-1, j+1},
\end{aligned}
$$

and, finally,

$$
\begin{aligned}
\left\langle x^{j}, L_{n}^{(\alpha, N)}\right\rangle= & \int_{0}^{\infty} x^{j} L_{n}^{(\alpha, N)}(x) x^{\alpha} e^{-x} d x+\left.N j !\left[L_{n}^{(\alpha, N)}(x)\right]^{(j)}\right|_{x=0} \\
= & A_{n, 0} \cdot 0+A_{n, 1} \cdot 0+A_{n, 2} \cdot 0+\cdots+A_{n, j} \cdot 0+A_{n, j+1} \cdot I_{0, j+1} \\
& +N \Gamma(j+1)(-1)^{j} \sum_{k=0}^{j+1} A_{n, k} L_{n-k-j}^{(\alpha+k+j)}(0) .
\end{aligned}
$$


Using the formula of $I_{k, i}$, the identity (5.1.7) in Szegö's book [13], and the expression of $A_{n, k}, 0 \leq k \leq j+1$, given in Theorem 1, our statement follows.

Notice that a different expression for the connection formula appears in Theorem 3.1 in [12]. Using the structure relation for Laguerre polynomials and after cumbersome computations, (2.3) can be deduced. Our proof is more simple as well as here the connection coefficients are explicitly given.

Lemma 1. For $n>j$ and each $\alpha>-1$, the inequalities

$$
A_{n, 0} \geq 1,(-1)^{k} A_{n, k} \geq 0, k=1,2, \ldots, j+1
$$

hold.

Proof. The positivity of the numbers $(-1)^{k} A_{n, k}, 1 \leq k \leq j+1$ is obvious. To prove that $A_{n, 0}-1 \geq 0$, notice that

$$
\left(\begin{array}{c}
n+\alpha \\
n-j-\nu
\end{array}\right)\left(\begin{array}{c}
n-\nu \\
j+1-\nu
\end{array}\right)-\left(\begin{array}{c}
n+\alpha \\
n-j-\nu-1
\end{array}\right)\left(\begin{array}{c}
n-\nu-1 \\
j-\nu
\end{array}\right) \geq 0
$$

holds for $n>j, \alpha>-1$, and $\nu=1,3,5, \ldots, j$ or $j-1$ depending on the parity of $j$.

\section{THE ZEROS}

From (2.3), $L_{n}^{(\alpha, N)}(x)$ can be written as

$$
L_{n}^{(\alpha, N)}(x)=\left(1+\widetilde{A}_{n, 0} N\right) L_{n}^{(\alpha)}(x)+\sum_{k=1}^{j+1} N \widetilde{A}_{n, k} L_{n-k}^{(\alpha+k)}(x),
$$

where $\widetilde{A}_{n, 0}=\left(A_{n, 0}-1\right) / N$ and $\widetilde{A}_{n, k}=A_{n, k} / N, k=1, \ldots, j+1$. Now, we introduce the polynomial

$$
F_{n, \alpha, l}(x)=-\sum_{k=0}^{j+1} \widetilde{A}_{n, k} L_{n-k}^{(\alpha+k)}(x) .
$$

Then, (3.5) reads

$$
L_{n}^{(\alpha, N)}(x)=L_{n}^{(\alpha)}(x)-N F_{n, \alpha, l}(x) .
$$

Let us denote by $x_{n, k}^{N}(\alpha), x_{n, k}(\alpha)$, and $\zeta_{n, k}(\alpha)$ the zeros of $L_{n}^{(\alpha, N)}(x), L_{n}^{(\alpha)}(x)$, and $F_{n, \alpha, l}(x)$, respectively, arranged in a decreasing order. It was proved in $[6$, Theorem 3 , that at most one of the zeros of $L_{n}^{(\alpha, N)}(x)$ is located outside $(0, \infty)$. Notice that this is a particular case of Theorem 4.1 in 12 .

In the present work, we will give explicitly the value $N_{0}$ of the mass such that for $N>N_{0}$ this situation occurs; i.e., the least zero is negative. Moreover, in [6], it was shown that $x_{n, n}^{N}(\alpha)<x_{n, n}(\alpha)<\cdots<x_{n, 1}^{N}(\alpha)<x_{n, 1}(\alpha)$ (see also Theorem 4.3 in [12]). In such a sense, here we complete this result proving that the zeros $x_{n, k}^{N}(\alpha)$ and $x_{n, k}(\alpha)$ interlace with the zeros $\zeta_{n, k}(\alpha)$.

Theorem 2. For every $n>j$ and each $\alpha>-1$, the inequalities

$$
\zeta_{n, n}(\alpha)<x_{n, n}^{N}(\alpha)<x_{n, n}(\alpha)<\cdots<\zeta_{n, 1}(\alpha)<x_{n, 1}^{N}(\alpha)<x_{n, 1}(\alpha)
$$


hold. Moreover, the smallest zero $x_{n, n}^{N}(\alpha)$ satisfies

$$
\begin{aligned}
& x_{n, n}^{N}(\alpha)>0, \text { for } N<N_{0}, \\
& x_{n, n}^{N}(\alpha)=0, \text { for } N=N_{0}, \\
& x_{n, n}^{N}(\alpha)<0, \text { for } N>N_{0},
\end{aligned}
$$

where

$$
N_{0}=N_{0}(n, \alpha, j)=\frac{L_{n}^{(\alpha)}(0)}{F_{n, \alpha, l}(0)}=\frac{\left(\begin{array}{c}
n+\alpha \\
n
\end{array}\right)}{-\sum_{k=0}^{j+1} \widetilde{A}_{n, k}\left(\begin{array}{c}
n+\alpha \\
n-k
\end{array}\right)} .
$$

Proof. Observe that the leading coefficients of $L_{n}^{(\alpha, N)}(x), L_{n}^{(\alpha)}(x)$, and $F_{n, \alpha, l}(x)$ are equal to

$$
\frac{(-1)^{n} A_{n, 0}}{n !}, \quad \frac{(-1)^{n}}{n !} \text { and } \frac{(-1)^{n-1} \widetilde{A}_{n, 0}}{n !},
$$

respectively. Since (see [6, Thm. 4]) $x_{n, n}^{N}(\alpha)<x_{n, n}(\alpha)<\cdots<x_{n, 1}^{N}(\alpha)<x_{n, 1}(\alpha)$, then, using the connection formula (3.7), we get

$$
\operatorname{sign}\left(L_{n}^{(\alpha, N)}\left(x_{n, k}(\alpha)\right)\right)=-\operatorname{sign}\left(F_{n, \alpha, l}\left(x_{n, k}(\alpha)\right)\right)=(-1)^{n-k+1}
$$

and

$$
\operatorname{sign}\left(L_{n}^{(\alpha)}\left(x_{n, k}^{N}(\alpha)\right)\right)=\operatorname{sign}\left(F_{n, \alpha, l}\left(x_{n, k}^{N}(\alpha)\right)\right)=(-1)^{n-k},
$$

for $k=1, \ldots, n$. Thus, the interlacing property (3.8) follows. In order to investigate the location of $x_{n, n}^{N}(\alpha)$ with respect to the origin, it suffices to observe that $L_{n}^{(\alpha, N)}(0)=0$ if and only if $N=N_{0}$.

In order to show the behavior of the least zero $x_{n, n}^{N}(\alpha)$ of the Laguerre-Sobolevtype orthogonal polynomials, we will do some numerical computations using the Mathematica software. We present, for $n, j$, and $\alpha$ fixed, a table that shows the behavior of $x_{n, n}^{N}(\alpha)$ with respect to $N$. In particular, for $N>N_{0}$, the least zero is negative.

For the case $j=1, N_{0}$ reduces to

$$
N_{0}=N_{0}(n, \alpha, 1)=\frac{\Gamma(n-1) \Gamma(\alpha+2) \Gamma(\alpha+4)}{\Gamma(n+\alpha+2)} .
$$

Then, for $n=2,3$ and $\alpha=-1 / 2,1,5$ we get

\begin{tabular}{|c|c|c|c|c|c|}
\hline$N$ & $x_{2,2}^{N}(-1 / 2)$ & $N$ & $x_{2,2}^{N}(1)$ & $N$ & $x_{2,2}^{N}(5)$ \\
\hline $1 / 2$ & 0.115964 & $3 / 2$ & 0.271499 & 710 & 0.0419159 \\
\hline$N_{0}=\sqrt{\pi} / 2$ & 0 & $N_{0}=2$ & 0 & $N_{0}=720$ & 0 \\
\hline 1 & -0.0313955 & $5 / 2$ & -0.230139 & 730 & -0.0414199 \\
\hline
\end{tabular}

\begin{tabular}{|c|c|c|c|c|c|}
\hline$N$ & $x_{3,3}^{N}(-1 / 2)$ & $N$ & $x_{3,3}^{N}(1)$ & $N$ & $x_{3,3}^{N}(5)$ \\
\hline $1 / 4$ & 0.00211646 & $1 / 5$ & 0.407703 & 79 & 0.0251697 \\
\hline$N_{0}=\sqrt{\pi} / 7$ & 0 & $N_{0}=2 / 5$ & 0 & $N_{0}=80$ & 0 \\
\hline $1 / 2$ & -0.133233 & $3 / 5$ & -0.275762 & 81 & -0.0248324 \\
\hline
\end{tabular}


For the case $j=2, N_{0}$ reduces to

$$
N_{0}=N_{0}(n, \alpha, 2)=\frac{\Gamma(n-2) \Gamma(\alpha+3) \Gamma(\alpha+6)}{2[n(\alpha+4)-(\alpha+2)] \Gamma(n+\alpha+2)} .
$$

Thus, for $n=3$, and $\alpha=-1 / 2, \alpha=1, \alpha=5$ we obtain

\begin{tabular}{|c|c|c|c|}
\hline$N$ & $x_{3,3}^{N}(-1 / 2)$ & $N$ & $x_{3,3}^{N}(1)$ \\
\hline $1 / 4$ & 0.0715899 & 1 & 0.415775 \\
\hline$N_{0}=3 \sqrt{\pi} / 16$ & 0 & $N_{0}=3 / 2$ & 0 \\
\hline $1 / 3$ & -0.00100291 & 2 & -0.450761 \\
\hline
\end{tabular}

\begin{tabular}{|c|c|}
\hline$N$ & $x_{3,3}^{N}(5)$ \\
\hline 1259 & 0.00317496 \\
\hline$N_{0}=1260$ & 0 \\
\hline 1261 & -0.00317424 \\
\hline
\end{tabular}

For the case $j=3, N_{0}$ reduces to

$$
\begin{aligned}
N_{0} & =N_{0}(n, \alpha, 3) \\
& =\frac{\Gamma(n-3) \Gamma(\alpha+4) \Gamma(\alpha+8)}{3\left[n^{2}(\alpha+5)(\alpha+6)-3 n(\alpha+4)(\alpha+5)+2(\alpha+2)(\alpha+3)\right] \Gamma(n+\alpha+2)} .
\end{aligned}
$$

Thus, for $n=4$, and $\alpha=-1 / 2, \alpha=1, \alpha=5$ we obtain

\begin{tabular}{|c|c|c|c|}
\hline$N$ & $x_{4,4}^{N}(-1 / 2)$ & $N$ & $x_{4,4}^{N}(1)$ \\
\hline $1 / 10$ & 0.102777 & 1 & 0.322548 \\
\hline$N_{0}=5 \sqrt{\pi} / 48$ & 0 & $N_{0}=4 / 3$ & 0 \\
\hline $2 / 10$ & -0.0346506 & $5 / 3$ & -0.41383 \\
\hline
\end{tabular}

\begin{tabular}{|c|c|}
\hline$N$ & $x_{4,4}^{N}(5)$ \\
\hline 2239 & 0.00200886 \\
\hline$N_{0}=2240$ & 0 \\
\hline 2241 & -0.00200899 \\
\hline
\end{tabular}

In the next result, we obtain the monotonicity of the zeros $x_{n, k}^{N}(\alpha)$ with respect to $N$ as well as their convergence when $N$ tends to infinity to the zeros $\zeta_{n, k}(\alpha)$ with a speed of convergence of order $1 / N$. For the proof of these statements, we need the following lemma concerning the behavior of the zeros of linear combinations of two polynomials with interlacing zeros.

Lemma 2. Let $h_{n}(x)=(-1)^{n} a\left(x-x_{1}\right) \cdots\left(x-x_{n}\right)$ and $g_{n}(x)=(-1)^{n-1} b(x-$ $\left.\zeta_{1}\right) \cdots\left(x-\zeta_{n}\right)$ be polynomials with real and interlacing zeros,

$$
\zeta_{n}<x_{n}<\cdots<\zeta_{1}<x_{1},
$$

where $a$ and $b$ are real positive constants. Then, for any real constant $c>0$, the polynomial

$$
f(x)=h_{n}(x)-c g_{n}(x)
$$


has $n$ real zeros $\eta_{n}<\eta_{n-1}<\cdots<\eta_{1}$ which interlace with both the zeros of $h_{n}(x)$ and $g_{n}(x)$ in the following form:

$$
\zeta_{n}<\eta_{n}<x_{n}<\cdots<\zeta_{1}<\eta_{1}<x_{1} .
$$

Moreover, each $\eta_{k}$ is a decreasing function of $c$ and, for each $k=1, \ldots, n$,

$$
\lim _{c \rightarrow \infty} \eta_{k}=\zeta_{k} \text { and } \lim _{c \rightarrow \infty} c\left[\eta_{k}-\zeta_{k}\right]=\frac{h_{n}\left(\zeta_{k}\right)}{g_{n}^{\prime}\left(\zeta_{k}\right)} .
$$

Recent results concerning zeros of linear combinations of orthogonal polynomials have been used in 4 and 5 in order to analyze monotonicity and the asymptotics for the zeros of some class of orthogonal polynomials. We omit here the proof of the above lemma (see [3, Lemma 1] and [5, Lemma 3]).

Theorem 3. For every $n>j$ and each $\alpha>-1$, the zeros $x_{n, k}^{N}(\alpha)$ are decreasing functions of $N$. Moreover,

$$
\lim _{N \rightarrow \infty} x_{n, k}^{N}(\alpha)=\zeta_{n, k}(\alpha)
$$

and

where

$$
\lim _{N \rightarrow \infty} N\left[x_{n, k}^{N}(\alpha)-\zeta_{n, k}(\alpha)\right]=g_{n, k, l}(\alpha), \quad k=1, \ldots, n,
$$

$$
g_{n, k, l}(\alpha)=\frac{L_{n}^{(\alpha)}\left(\zeta_{n, k}(\alpha)\right)}{F_{n, \alpha, l}^{\prime}\left(\zeta_{n, k}(\alpha)\right)} .
$$

Proof. This is an immediate consequence of (3.7), the inequalities (3.8), and Lemma 2.

\section{ACKNOWLEDGEMENTS}

We thank the referee for suggestions in order to improve the presentation of the manuscript. In particular, we have emphasized the connections of our results with some previous ones done in [12] (for section 2) and [3] (for section 3), where quite different approaches are presented. The work of the first author has been supported by Dirección General de Investigación, Ministerio de Ciencia e Innovación of Spain, grant MTM2009-12740-C03-01. The work of the second author has been supported by FAPESP under grant 07/02854-6. This manuscript was finished during the second author's stay at the Departmento de Matemáticas, Universidad Carlos III de Madrid, in the framework of a joint project of Dirección General de Investigación, Ministerio de Educación y Ciencia of Spain and the Brazilian Science Foundation CAPES, Project CAPES/DGU 160/08.

\section{REFERENCES}

[1] R. Alvarez-Nodarse and J. J. Moreno Balcázar, Asymptotic properties of generalized Laguerre orthogonal polynomials, Indag. Math. (N.S.) 15 (2004), 151-165. MR2071854(2005e:33003)

[2] H. Bavinck, Differential operators having Sobolev type Laguerre polynomials as eigenfunctions, Proc. Amer. Math. Soc. 125 (1997), 3561-3567. MR.1422848 (98b:33019)

[3] C. F. Bracciali, D. K. Dimitrov, and A. Sri Ranga, Chain sequences and symmetric generalized orthogonal polynomials, J. Comput. Appl. Math. 143 (2002), 95-106. MR.1907785 (2003f:33007)

[4] D. K. Dimitrov, F. Marcellán, and F. R. Rafaeli, Monotonicity of zeros of Laguerre-Sobolevtype orthogonal polynomials, J. Math. Anal. Appl. 368 (2010), 80-89. MR2609260

[5] D. K. Dimitrov, M. V. Mello, and F. R. Rafaeli, Monotonicity of zeros of Jacobi-Sobolev type orthogonal polynomials, Appl. Numer. Math. 60 (2010), 263-276. MR2602677 
[6] H. Dueñas and F. Marcellán, The Laguerre-Sobolev-type orthogonal polynomials, J. Approx. Theory 162 (2010), 421-440. MR.2581392

[7] H. Dueñas and F. Marcellán, The Laguerre-Sobolev-type orthogonal polynomials. Holonomic equation and electrostatic interpretation, Rocky Mount. J. of Math. (2011). In press.

[8] R. Koekoek and H. G. Meijer, A generalization of Laguerre polynomials, SIAM J. Math. Anal. 24 (1993), 768-782. MR.1215437 (94b:33007)

[9] J. Koekoek, R. Koekoek, and H. Bavinck, On differential equations for Sobolev-type Laguerre polynomials, Trans. Amer. Math. Soc. 350 (1998), 347-393. MR1433121 (98d:33003)

[10] F. Marcellán and J. J. Moreno-Balcázar, Asymptotics and zeros of Sobolev orthogonal polynomials on unbounded supports, Acta Appl. Math. 94 (2006), 163-192. MR 2273888 (2007i:42002)

[11] F. Marcellán and A. Ronveaux, On a class of polynomials orthogonal with respect to a discrete Sobolev inner product, Indag. Math. (N.S.) 1 (1990), 451-464. MR1106092 (92f:42029)

[12] H. G. Meijer, Zero distribution of orthogonal polynomials in a certain discrete Sobolev space, J. Math. Anal. Appl. 172 (1993), 520-532. MR1201003 (94a:42027)

[13] G. Szegö, Orthogonal Polynomials, Amer. Math. Soc. Coll. Publ., Vol. 23, 4th ed., Amer. Math. Soc., Providence, RI, 1975. MR0372517 (51:8724)

Departamento de Matemáticas, Escuela Politécnica Superior, Universidad Carlos III De Madrid, 28911 Leganés, Spain

Instituto de Matemática, Estatística e Computação Científica, Universidade Estadual de Campinas, São Paulo, Brazil

Current address: Departamento de Matemática, Estatística e Computação/FCT, Universidade Estadual Paulista-UNESP, 19060-900 Presidente Prudente, São Paulo, Brazil 